\title{
A novel somatic $B R C A 2$ point mutation in a metastatic pancreatic cancer patient: a case report
}

\author{
Deqiang Wang ${ }^{1}$, Ruting Guan², Qing Tao ${ }^{1}$, Sisi Liu², Man Yu³ and Xiaoqin $\mathrm{Li}^{3}{ }^{*}$ (D)
}

\begin{abstract}
Background: In addition to ovarian and breast cancers, loss-of-function mutations in BRCA1 and BRCA2 genes are also linked to an increased risk of pancreatic cancer, with $\sim 4$ to $7 \%$ of pancreatic cancer patients harboring germline $B R C A$ mutations. Most BRCA alterations in pancreatic cancer are frame-shifting indels, stop-gain, and splice-site mutations, but single nucleotide substitutions are rare. Recent studies demonstrated a significant progression-free survival (PFS) benefit from maintenance olaparib, a poly (ADP-ribose) polymerase (PARP) inhibitor administered to patients with germline BRCA mutations and metastatic pancreatic cancer.
\end{abstract}

Case presentation: Here, we report a metastatic pancreatic cancer case who harbored a novel somatic BRCA2 c.6944T > C (p. I2315T) point mutation. After 6 weeks first-line chemotherapy, the patient was refractory to treatment and had a progressive disease. Due to the novel nonsynonymous BRCA2 point mutation, we decided to change the strategy by administering olaparib. The patient benefited from olaparib therapy and achieved a PFS of $\sim 6.5$ months.

Conclusions: We describe a patient carrying a novel somatic BRCA2 p. I2315T point mutation, which is first reported in metastatic pancreatic cancer. This case report indicates that a gene mutation-based strategy should be considered in the clinic to provide more effective treatment.

Keywords: BRCA2, Olaparib, Metastatic pancreatic cancer, PARP inhibitor, Somatic mutation

\section{Background}

Pancreatic cancer is the 7th leading cause of cancerrelated deaths worldwide, with a 5-year survival rate of $<5 \%$ [1]. This devastating malignancy is generally diagnosed at metastatic stages in the clinic, indicative of its late detection and biological aggressiveness [1]. Tremendous efforts are ongoing with the aims of discovering early diagnostic markers and novel therapeutic avenues for pancreatic cancer; however, progress is remarkably hindered by the complicated heterogeneity within and between patient tumors.

\footnotetext{
*Correspondence: lixiaoqin9966@126.com

1 Department of Medical Oncology, Affiliated Hospital of Jiangsu

University, Zhenjiang, Jiangsu, China

Full list of author information is available at the end of the article
}

$B R C A 1$ and $B R C A 2$ are essential players involved in homologous recombination repair of double-strand deoxyribonucleic acid (DNA) breaks [2]. BRCA inactivation due to somatic mutations or $B R C A 1$ promoter methylation have been observed in various cancer types, including a small subgroup of metastatic pancreatic cancer [3-5]. Most BRCA alterations in pancreatic cancer are frame-shifting indels, stop-gain, and splice-site mutations, whereas single nucleotide substitutions are rarely identified $[2,4,5]$.

A large body of evidence showed that the poly (ADPribose) polymerase (PARP) inhibitors are synthetically lethal in BRCA-mutated tumors with DNA repair defects and displayed potent anti-tumor activity when combined with DNA-damaging agents. Thus, patients carrying germline mutations in $B R C A$ or the various patterns of 
somatic $B R C A$ mutations that could result in the inactivation of $B R C A$ are sensitive to PARP inhibitors [6].

A recent phase 3 POLO (Pancreas Cancer Olaparib Ongoing) trial demonstrated that the administration of a PARP inhibitor, olaparib, as a maintenance therapy significantly prolonged the median progression-free survival (PFS) of patients with germline BRCA mutations and metastatic pancreatic cancer who had not progressed during platinum-based chemotherapy compared to the placebo arm (7.4 months vs. 3.8 months) [7]. More importantly, olaparib treatment did not compromise health-related quality of life in those patients [8]. However, PARP inhibitor resistance is common due to homologous recombination repair restoration (HRR), epigenetic modification, reversion mutations, restoration of ADP-ribosylation (PARylation), and pharmacological alteration [9].

In this report, we present the case of a metastatic pancreatic cancer patient who had progressive disease (PD) following chemotherapy with gemcitabine and nab-paclitaxel. Mutational profiling analysis using targeted nextgeneration sequencing (NGS) revealed that the patient carried a novel somatic BRCA2 c.6944T >C (p. I2315T) point mutation. Olaparib was thereby administered in combination with a modified FOLFIRINOX regimen or as a monotherapy. The patient showed a significant response to this treatment strategy and exhibited stable disease and a PFS of $\sim 6.5$ months was observed.

\section{Case presentation}

A 57-year-old Chinese male with no obvious symptoms was admitted to the hospital due to the identification of a low-density shadow in the liver during his physical examination. In January 2019, a computed tomography (CT) scan revealed a $5.0 \times 3.0 \mathrm{~cm}$ tumor in the uncinate process of the pancreas with hepatic metastases (Fig. 1a). The patient had a family history of cancer: his mother was diagnosed with stomach cancer at the age of 65 years and his father had lung cancer. According to the National Comprehensive Cancer Network (NCCN) Guidelines, the patient first received gemcitabine treatment at a dose of $1.4 \mathrm{~g}$ once per week, and nab-paclitaxel at a dose of 200 mg every 2 weeks as a first-line chemotherapy in January 2019 (Fig. 1b). Unfortunately, a CT re-evaluation at 6 weeks post-treatment showed progressive enlargement of both pancreatic and hepatic lesions (Fig. 1a), accompanied with a marked elevation of serum carcinoembryonic antigen (CEA, $270.35 \mathrm{ng} / \mathrm{mL}$ ) and carbohydrate antigen 19-9 (CA19-9, $352.56 \mathrm{U} / \mathrm{mL}$ ) levels (Fig. 1c); thus, suggesting that the patient was refractory to treatment and had a PD.

To determine a more effective and appropriate targeted therapy, we performed a targeted NGS analysis of 425 cancer-related genes on the patient's plasma and tumor tissue biopsy samples. The cancer mutation panel test revealed multiple deleterious somatic mutations, such as the driver mutations, KRAS Q61R $(\mathrm{c} .182 \mathrm{~A}>\mathrm{G})$, TP53 R110del (c.329_331delGTC), and APC S1465RfsX9 (c.4393_4394dupAG), and copy number gain of several genes (Table 1). Interestingly, we also observed a novel nonsynonymous $B R C A 2$ c.6944T $>C$ (p. I2315T) point mutation with a mutant allele frequency (MAF) of $28.0 \%$ in the plasma and $39.5 \%$ in the tumor biopsy specimens. The novel BRCA2 point mutation was not detected in white blood cells, and thus, it was confirmed as a somatic mutation.

Given the clinical efficacy of olaparib in treating $B R C A$ -mutated advanced solid tumors, we decided to revise the therapeutic strategy by administering olaparib at a dose of $150 \mathrm{mg}$ twice per day in combination with a modified FOLFIRINOX regimen (irinotecan at a dose of $100 \mathrm{mg}$ on days 1,8 and 15; oxaliplatin at a dose of $150 \mathrm{mg}$ on days 1 and 15; S-1 at a dose of $40 \mathrm{mg}$ twice per day from day 1 to day 14) for one cycle beginning in March 2019 (Fig. 1b). However, due to the occurrence of adverse events (e.g., diarrhea, general weakness, fatigue, and loss of appetite) and myelosuppression, including grade 2-3 leukopenia and thrombocytopenia, chemotherapy was not given on day 15 and the frequency of irinotecan was reduced to only be administered on days 1 and 15 in the subsequent treatment. Additionally, megestrol acetate and granulocyte-colony stimulating factor (G-CSF) were prescribed to increase appetite and promote the recovery of myelosuppression. In May 2019, a reduction in the size of both pancreatic and hepatic lesions was revealed by the CT scan, while the levels of the serum tumor biomarkers, CEA $(63.38 \mathrm{ng} / \mathrm{mL})$ and CA19-9 $(40.53 \mathrm{U} / \mathrm{mL})$ were considerably reduced (Fig. 1a, c). Olaparib and modified FOLFIRINOX were thus continued with irinotecan and oxaliplatin being administered only on day 1 (Fig. 1b). After 2 weeks of treatment, CEA and CA19-9 levels decreased to $32.69 \mathrm{ng} / \mathrm{mL}$ and $25.57 \mathrm{U} / \mathrm{mL}$, respectively (Fig. 1c). A CT scan performed in June 2019 showed a significant size reduction in the primary and metastatic tumors, which indicated a partial response (PR, Fig. 1a). Since the patient experienced severe marrow suppression during treatment, only olaparib monotherapy was administered since July 2019 to reduce adverse effects (Fig. 1b). Stable disease (SD) was subsequently observed in August 2019 (Fig. 1a). In September 2019, aside from an increase in CEA and CA19-9 levels (Fig. 1c), laboratory blood tests demonstrated that the patient underwent acquired granulocytopenia, anemia, and thrombocytopenia. Consequently, G-CSF, thrombopoietin, and erythropoietin were administered for symptom management. After olaparib treatment for another 1.5 months, PD was 

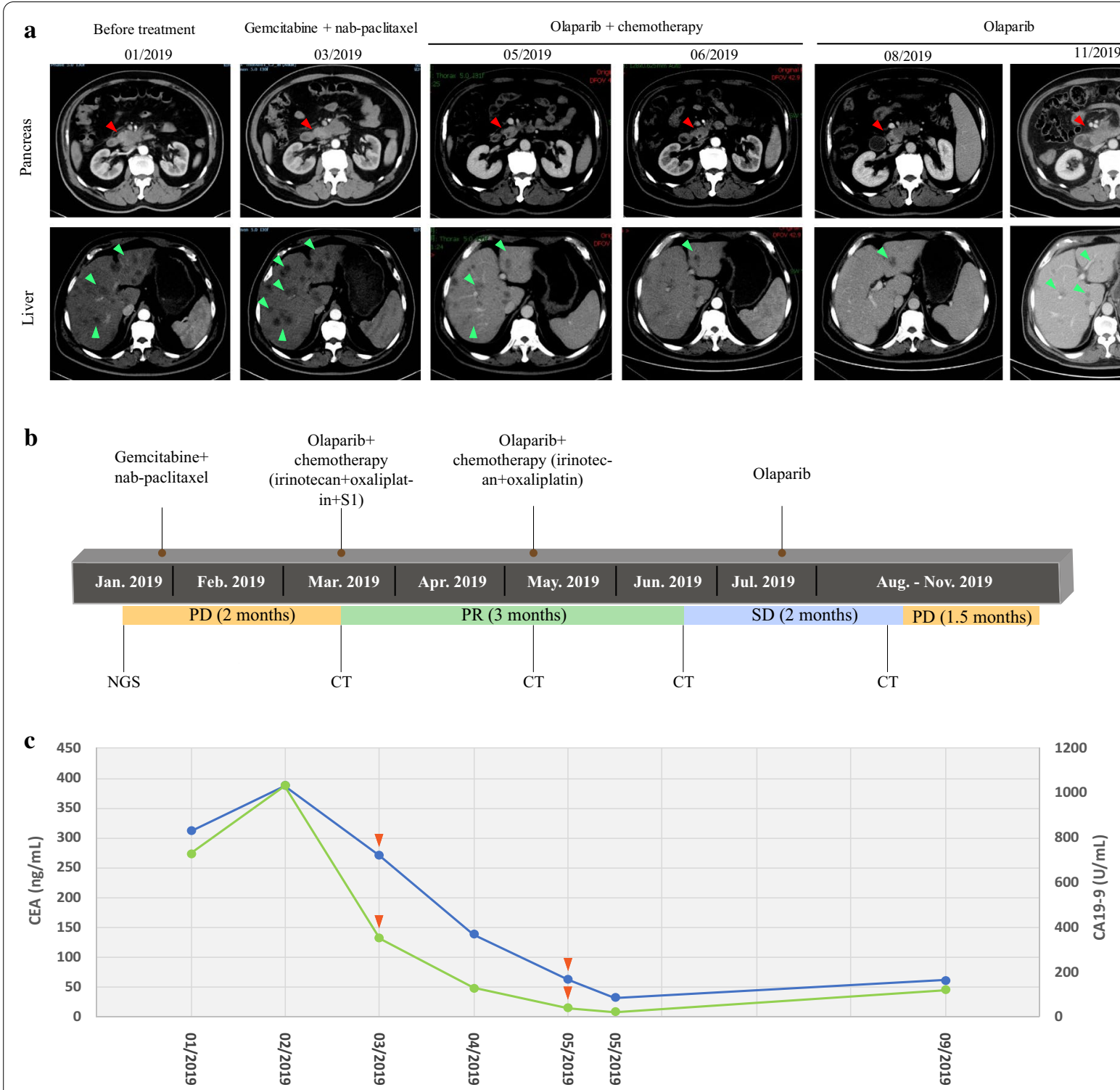

Fig. 1 CT images and measurements of serum tumor biomarkers during the course of treatment. a Serial CT scans demonstrated a reduction in the size of the pancreatic (red arrows) and metastatic hepatic lesions (green arrows) following olaparib therapy. $\mathbf{b}$ A timeline indicating the application of different therapeutic strategies and the patient's response. c Measurements of serum CEA and CA19-9 levels at different treatment times. Blue line: CEA; green line: CA19-9; orange arrows: time points of the CT scans. PD progressive disease, PR partial response, SD stable disease

indicated by a CT scan (Fig. 1a). The patient achieved a PFS for $~ 6.5$ months following olaparib combination and monotherapy.

\section{Discussion and conclusions}

Neoplastic cells lacking a functional homologous recombination repair system, such as those carrying $B R C A$ mutations, are sensitive to PARP inhibition through accumulated DNA damage via multiple mechanisms [6]. As a new therapeutic concept, maintenance olaparib has shown promising results in the treatment of germline $B R C A$-mutated breast, ovarian, and metastatic pancreatic cancer $[7,10,11]$. To date, the majority of $B R C A 2$ mutations identified in pancreatic cancer were frame-shifting indels (e.g., c.6174delT, c.6158insT) and splice-site mutations. In contrast, single point mutations have been rarely reported $[4,5,12]$. Mesman [13] recently assessed the potential pathogenic impact of a large set of BRCA2 missense variants using a mouse embryonic stem cell (mESC)-based functional assay, and found that BRCA2 missense mutations, such as c.93G $>\mathrm{T}$ (p. W31C) and c.8351G > A (p. R2784Q), 
Table 1 Genetic alterations detected in the patient's plasma and tumor biopsy specimens

\begin{tabular}{|c|c|c|c|c|}
\hline Genes & Alternations & Nucleotide change & MAF (plasma) & MAF (tumor) \\
\hline$B R C A 2$ & p. $12315 T$ & c.6944T >C & $28 \%$ & $39.5 \%$ \\
\hline KRAS & p. Q61R & c. $182 A>G$ & $27.4 \%$ & $43.2 \%$ \\
\hline TP53 & p. R110del & c.329_331delGTC & $37.4 \%$ & $81.6 \%$ \\
\hline$A P C$ & p. S1465RfsX9 & c.4393_4394dupAG & $26.7 \%$ & $71.7 \%$ \\
\hline CCNE1 & Gene amplification & NA & 16.0-fold & 27.4-fold \\
\hline CCNE1 & $\begin{array}{l}\text { IGR (downstream UQCRFS1) CCNE1 } \\
\text { fusion }\end{array}$ & NA & $0.1 \%$ & - \\
\hline PIK3CA & Gene amplification & NA & - & 1.9-fold \\
\hline PKHD1 & p. R909X truncation & $c .2725 C>T$ & $4.9 \%$ & $16.9 \%$ \\
\hline $\mathrm{SO} 2$ & Gene amplification & NA & - & 2.0-fold \\
\hline STMN1 & Gene amplification & NA & - & 1.8-fold \\
\hline TERC & Gene amplification & NA & - & 1.9-fold \\
\hline TUBB3 & Gene amplification & NA & - & 2.2-fold \\
\hline
\end{tabular}

-, not detectable; NA, not applicable; MAF, mutant allele frequency. Gene amplification was presented as the relative fold change to normal controls

were able to increase susceptibility to PARP inhibitor treatment.

The patient in our report harbored an unreported BRCA2 point mutation [c.6944T > C (p. I2315T)] located in exon 13 of $B R C A 2$ between the G-CSF. Different germline BRCA2 mutations (e.g., p. I2315V, p. I2315L) at the same site have been documented with unknown functional significance [14, 15]. Although the underlying molecular mechanism(s) by which this novel BRCA2 p. I2315T mutation impairs DNA repair and sensitizes tumor cells to PARP inhibition remains to be elucidated. We postulate that it may be related to a change in the polarity of amino acid residues since isoleucine (I), valine (V) and leucine (L) are all non-polar and hydrophobic, while threonine $(\mathrm{T})$ is hydrophilic. Thus, we hypothesize that the p. I2315T mutation is likely to cause a structural abnormality in the $B R C A 2$ protein, which results in defective DNA double strand break (DSB) repair by homologous recombination (HR) and its sensitivity to olaparib. To test this hypothesis, we attempted to generate a three dimensional (3D) structural model of the $B R C A 2$ p. I2315T mutation, but failed due to the absence of an established crystal structure of the full-length human $B R C A 2$ due to its size and segmental nature [16].

In summary, we report a metastatic pancreatic cancer patient carrying a novel somatic BRCA2 p. I2315T point mutation. Furthermore, advances in NGS technology have provided a solid basis for precise detection of well-known driver mutations, and rare or novel mutations. Thus, NGS may provide clinicians with invaluable information (e.g., BRCA1/2 status of tumors) that can be leveraged for therapeutic decision making, and perform better evaluations of patients' responses during the course of treatment.

\section{Abbreviations \\ PARP: Poly(ADP-ribose) polymerase; PFS: Progression-free survival; PD: Progres- sive disease; DNA: Deoxyribonucleic acid; POLO: Pancreas cancer olaparib ongoing; CT: Computed tomography; NCCN: The National Comprehensive Cancer Network; CEA: Carcinoembryonic antigen; CA19-9: Carbohydrate anti- gen 19-9; NGS: Next-generation sequencing; MAF: Mutant allele frequency; FOLFIRINOX: FOL-folinic acid, F-fluorouracil, IRIN-irinotecan, OX-oxaliplatin; G-CSF: Granulocyte-colony stimulating factor; PR: Partial response; mESC: Mouse embryonic stem cell; DSBs: Double strand breaks; HR: Homologous recombination; 3D: Three dimensional.}

\section{Acknowledgements}

We would like to thank the patient for providing written informed consent for publication.

\section{Authors' contributions}

XL conceptualized and designed the manuscript. QT and DW carried out patient clinical management, sample collection and drafted the manuscript. RG analyzed the data. SL and MY revised the manuscript. All authors read and approved the final manuscript.

\section{Funding}

Supported by the Key Project of Zhenjiang City for Health Science and Technology (SH2016038 and SH2019046), and the Project of Young Medical Talents in Jiangsu Province (QNRC2016829), China. The funding body did not have any role in the study design, data collection and analysis, decision to publish, or preparation of the manuscript.

\section{Availability of data and materials}

The datasets generated and/or analysed during the current study are not publicly available in order to protect participant confidentiality but are available from the corresponding author on reasonable request.

\section{Ethics approval and consent to participate}

The study protocol was approved by the ethical committee of the Affiliated Hospital of Jiangsu University. Written informed consent was provided by the patient. 


\section{Consent for publication}

Written informed consent was obtained from the patient for publication of the case and all accompanying tables/images. A copy of the written consent is available for review by the Editor of this journal.

\section{Competing interests}

Ruting Guan and Sisi Liu are the employees of Nanjing Geneseeq Technology Inc., China; Man Yu is an employee of Geneseeq Technology Inc., Canada. The remaining authors declare no conflicts of interest.

\section{Author details}

${ }^{1}$ Department of Medical Oncology, Affiliated Hospital of Jiangsu University, Zhenjiang, Jiangsu, China. ${ }^{2}$ Department of Research and Development, Nanjing Geneseeq Technology Inc., Nanjing, Jiangsu, China. ${ }^{3}$ Translational Medicine Research Institute, Geneseeq Technology Inc., Toronto, Canada.

Received: 20 November 2020 Accepted: 6 December 2020

Published online: 06 January 2021

\section{References}

1. Ducreux M, Seufferlein T, Van Laethem $J$, et al. Systemic treatment of pancreatic cancer revisited. Semin Oncol. 2019:46:28-38.

2. Gorodetska I, Kozeretska I, Dubrovska A. BRCA genes: the role in genome stability, cancer stemness and therapy Resistance. J Cancer. 2019;10:2109-27.

3. Welcsh PL, King MC. BRCA1 and BRCA2 and the genetics of breast and ovarian cancer. Hum Mol Genet. 2001;10:705-13.

4. Ghiorzo P. Genetic predisposition to pancreatic cancer. World J Gastroenterol. 2014:20:10778-89.

5. Holter S, Borgida A, Dodd A, et al. Germline BRCA mutations in a large clinic-based cohort of patients with pancreatic adenocarcinoma. J Clin Oncol. 2015;33(28):3124-9.

6. Faraoni I, Graziani G. Role of BRCA mutations in cancer treatment with poly(ADP-ribose) polymerase (PARP) inhibitors. Cancers. 2018;10:487.
7. Golan T, Hammel P, Reni M, et al. Maintenance olaparib for germline BRCA-mutated metastatic pancreatic cancer. N Engl J Med. 2019:381:317-27.

8. Hammel P, Kindler HL, Reni M, et al. Health-related quality of life in patients with a germline BRCA mutation and metastatic pancreatic cancer receiving maintenance olaparib. Ann Oncol. 2019;30:1959-68.

9. Li H, LiU Z-Y, Wu N, et al. PARP inhibitor resistance: the underlying mechanisms and clinical implications. Mol Cancer. 2020;19:107-107.

10. Robson M, Im S-A, Senkus E, et al. Olaparib for metastatic breast cancer in patients with a germline BRCA mutation. N Engl J Med. 2017;377:523-33.

11. Moore K, Colombo N, Scambia G, et al. Maintenance olaparib in patients with newly diagnosed advanced ovarian cancer. N Engl J Med. 2018;379:2495-505

12. Martinez-Useros J, Garcia-Foncillas J. The role of BRCA2 mutation status as diagnostic, predictive, and prognosis biomarker for pancreatic cancer. Biomed Res Int. 2016;2016:1869304.

13. Mesman RLS, Calleja F, Hendriks G, et al. The functional impact of variants of uncertain significance in BRCA2. Genet Med. 2019;21:293-302.

14. Juwle A, Saranath D. BRCA1/BRCA2 gene mutations/SNPs and BRCA1 haplotypes in early-onset breast cancer patients of Indian ethnicity. Med Oncol. 2012;29:3272-81.

15. Borg A, Haile RW, Malone KE, et al. Characterization of BRCA1 and BRCA2 deleterious mutations and variants of unknown clinical significance in unilateral and bilateral breast cancer: the WECARE study. Hum Mutat. 2010;31:E1200-40.

16. Shahid T, Soroka J, Kong E, et al. Structure and mechanism of action of the BRCA2 breast cancer tumor suppressor. Nat Struct Mol Biol. 2014;21:962-8.

\section{Publisher's Note}

Springer Nature remains neutral with regard to jurisdictional claims in published maps and institutional affiliations.
Ready to submit your research? Choose BMC and benefit from:

- fast, convenient online submission

- thorough peer review by experienced researchers in your field

- rapid publication on acceptance

- support for research data, including large and complex data types

- gold Open Access which fosters wider collaboration and increased citations

- maximum visibility for your research: over 100M website views per year

At BMC, research is always in progress.

Learn more biomedcentral.com/submissions 